Julia Maria Umińska1 ${ }^{1}$ Marek Koziński², Krzysztof Pstrągowski ${ }^{3}$, Przemysław Paciorek ${ }^{3}$, Tomasz Fabiszak ${ }^{3}$, Joanna Sikora ${ }^{1}$, Michał Marszałł ${ }^{4}$, Grzegorz Grześk ${ }^{1}$, Jacek Kubica ${ }^{3}$

${ }^{1}$ Department of Pharmacology and Therapeutics, Faculty of Medicine, Collegium Medicum, Nicolaus Copernicus University, Bydgoszcz, Poland

${ }^{2}$ Department of Principles of Clinical Medicine, Collegium Medicum, Nicolaus Copernicus University, Bydgoszcz, Poland

${ }^{3}$ Department of Cardiology and Internal Medicine, Collegium Medicum, Nicolaus Copernicus University, Bydgoszcz, Poland

${ }^{4}$ Department of Medicinal Chemistry, Collegium Medicum, Nicolaus Copernicus University, Bydgoszcz, Poland

\title{
Platelet reactivity during mild therapeutic hypothermia in patients with acute myocardial infarction treated with ticagrelor: study protocol of a single-centre study
}

\author{
Corresponding author: \\ Julia Maria Umińska \\ Department of Pharmacology and \\ Therapeutics \\ Collegium Medicum, \\ Nicolaus Copernicus University \\ Skłodowskiej-Curie Str. 9 \\ 85-094 Bydgoszcz, Poland \\ Tel. +48525853584 \\ E-mail: julia.m.kubica@gmail.com
}

Medical Research Journal 2016; Volume 1, Number 4, 115-119 10.5603/MRJ.2016.0021 Copyright @ 2016 Via Medica ISSN 2451-2591

\begin{abstract}
In summary, the available data on the antiplatelet efficacy of P2Y12 receptor inhibitors suggest their less potent and/or delayed effect in patients undergoing mild therapeutic hypothermia (MTH). However, previous studies do not explain the mechanisms of the impact of MTH on platelet function. Moreover, there is a lack of evidence of any relationship between the increased prevalence of thrombotic complications in MTH patients and the anti-platelet effect of P2Y12 receptor inhibitors.

We hypothesise that MTH may interfere with the absorption of ticagrelor and consequently results in significant changes in the pharmacokinetic and pharmacodynamic profile of this P2Y12 receptor inhibitor. It cannot be ruled out that the high initial level of platelet activation in MTH patients may additionally modify the pharmacodynamics of ticagrelor. Malabsorption may slow down the desired antiplatelet effect, while high levels of platelet activation may reduce the antiplatelet effect of ticagrelor.

The aim of this study is to verify whether the use of MTH after resuscitation in out-of-hospital cardiac arrest (OHCA) patients with STEMI (ST-segment elevation myocardial infarction) treated with primary percutaneous coronary intervention ( $\mathrm{pPCl}$ ), affects the anti-platelet effect of ticagrelor. Moreover, we attempt to elucidate the mechanisms of impaired effect of ticagrelor in MTH patients based on pharmacodynamic and pharmacokinetic measurements.

To achieve the aim of the study, we planned the following: 1) comparison of the pharmacokinetic and pharmacodynamic results obtained from the study population (MTH $+\mathrm{pPCl}+$ ticagrelor) with results obtained from a demographically and clinically comparable population of patients with STEMI treated with primary $\mathrm{PCl}$ and receiving ticagrelor ( $\mathrm{pPCl}+$ ticagrelor); and 2) analysis of the pharmacodynamic results in relation to the pharmacokinetic measurements in the target population.

Understanding the mechanisms standing behind the impact of MTH on the efficacy of platelet inhibition with P2Y12 inhibitors is pivotal in reducing the risk of thrombotic complications. The study is expected to provide information leading to improvement of the safety of MTH in STEMI patients with OHCA treated with $\mathrm{pPCl}$ and receiving ticagrelor.
\end{abstract}

Key words: platelet reactivity, ticagrelor, hypothermia

Med Res J 2016; 1 (4): 115-119 


\section{Introduction}

Platelet activation plays a key role in the pathophysiology of acute coronary syndromes, including myocardial infarction with ST-segment elevation (STEMI). Pharmacological platelet inhibition with P2Y12 receptor antagonists and acetylsalicylic acid is essential for the treatment and prevention of thrombotic complications in patients with STEMI undergoing primary percutaneous coronary intervention (pPCl) [1-3].

The results of the PLATO study showed that treatment with ticagrelor, a new potent inhibitor of the platelet $\mathrm{P} 2 \mathrm{Y} 12$ receptor, reduces the overall mortality and cardiovascular thrombotic events, as compared with clopidogrel, in patients with acute coronary syndromes (ACS), including STEMI [1]. The superiority of ticagrelor over clopidogrel in patients with ACS results from faster, more potent, and more uniform action of the former versus the latter of these drugs [1]. Ticagrelor currently holds a class I recommendation, level of evidence $B$ in patients with STEMI, both in the guidelines from the European Society of Cardiology (ESC) and from the American College of Cardiology/American Heart Association (ACCF/AHA) [2, 3].

Out-of-hospital cardiac arrest (OHCA) is the most frequent cause of sudden death in developed countries. OHCA survivors remaining in a coma despite restored cardiovascular function are saddled with a very high risk of death and serious neurological complications. The ESC and ACCF/AHA guidelines recommend the use of mild therapeutic hypothermia (MTH) in OHCA patients with myocardial infarction [2, 3]. According to the ESC guidelines [2], MTH is indicated early after resuscitation of cardiac arrest patients who are comatose or in deep sedation (Class of recommendation I, level of evidence B). Immediate angiography with a view to $\mathrm{pPCl}$ is recommended in patients with resuscitated cardiac arrest, whose ECG shows STEMI (class of recommendation I, level of evidence B), and it should be also considered in survivors of cardiac arrest without diagnostic ECG ST-segment elevation, but with a high suspicion of ongoing infarction. (Class of recommendation Ila, level of evidence B) [2]. According to the ACCF/AHA guidelines [3], MTH should be started as soon as possible in comatose patients with STEMI and out-of-hospital cardiac arrest caused by ventricular fibrillation (VF) or pulseless ventricular tachycardia (VT), including patients who undergo $\mathrm{pPCl}$ (class of recommendation I, level of evidence B). Immediate angiography and $\mathrm{pPCl}$ when indicated should be performed in resuscitated out-of-hospital cardiac arrest patients whose initial ECG shows STEMI (class of recommendation I, level of evidence B) [3].

Several studies, including our own $[4,5]$ as well as studies published by Casella et al. [6] and Dumas et al. [7], suggest that the combination of $\mathrm{PCl}$ and $\mathrm{MTH}$ is more effective than any of these methods used alone in the treatment of OHCA patients with ACS. However, there is a serious limitation of MTH in patients with acute myocardial infarction treated with $\mathrm{PCl}$ as described by Penela et al. [8]. They observed clinical resistance to clopidogrel with an extremely high incidence of acute stent thrombosis. In a small group of 11 MTH patients enrolled into the study, stent thrombosis occurred in five patients, while two other patients experienced other thrombotic complications [8]. This observation was not supported by any pharmacokinetic or pharmacodynamic data that could explain the mechanisms of thrombotic complications. In another study, Ibrahim et al. [9] compared the antiplatelet effect of clopidogrel, prasugrel, and ticagrelor 24 hours after loading dose in patients treated with and without hypothermia. The authors found a significantly higher incidence of unresponsiveness to the drug (PRI/VASP > 50\%) only among those patients undergoing MTH who received clopidogrel (82\% vs. $26 \%)$. However, the absolute values of PRI/VASP were also significantly higher in patients undergoing MTH and treated with ticagrelor. This study [9], suggesting less potent antiplatelet effect of all oral P2Y12 receptor inhibitors in patients undergoing MTH solely based on sparse pharmacodynamic data (one measurement after 24 hours), provides no information on pharmacokinetics, which would explain the mechanism of differences in platelet inhibition [9].

In summary, the available data on the antiplatelet efficacy of P2Y12 receptor inhibitors suggest their less potent and/or delayed effect in patients undergoing MTH. However, previous studies [8,9] do not explain the mechanisms of impact of MTH on platelet function. Moreover, there is a lack of evidence of any relationship between the increased prevalence of thrombotic complications in MTH patients and the anti-platelet effect of P2Y12 receptor inhibitors.

We hypothesise that MTH may interfere with the absorption of ticagrelor and consequently result in significant changes in the pharmacokinetic and pharmacodynamic profile of this $\mathrm{P} 2 \mathrm{Y} 12$ receptor inhibitor. It cannot be ruled out that the high initial level of platelet activation in MTH patients may additionally modify the pharmacodynamics of ticagrelor. Malabsorption may slow down the desired antiplatelet effect, while high levels of platelet activation may reduce the antiplatelet effect of ticagrelor.

The aim of this study is to verify whether the use of MTH after resuscitation in OHCA patients with STEMI treated with $\mathrm{pPCl}$ affects the anti-platelet effect of ticagrelor. Moreover, we attempt to elucidate the mechanisms of the impaired effect of ticagrelor in MTH patients based on the pharmacodynamic and pharmacokinetic measurements. 
To achieve the aim of the study, we have planned the following:

- comparison of the pharmacokinetic and pharmacodynamic results obtained from the study population $(\mathrm{MTH}+\mathrm{pPCl}+$ ticagrelor) with results obtained from a demographically and clinically comparable population of patients with STEMI treated with primary $\mathrm{PCl}$ and receiving ticagrelor ( $\mathrm{pPCl}+$ ticagrelor);

- analysis of the pharmacodynamic results in relation to the pharmacokinetic measurements in the target population.

\section{Methods}

This PK-PD, phase IV, single-centre, investigator-initiated, prospective, observational study will be conducted in accordance with the Declaration of Helsinki and Good Clinical Practice guidelines. The protocol of the study was approved by the Ethics Committee of The Nicolaus Copernicus University in Toruń, Collegium Medicum in Bydgoszcz (approval number KB 339/2015). Each patient with STEMI treated with $\mathrm{pPCl}$ without MTH will provide written, informed consent to participate in the study before enrolment. In this group of patients, additional blood sampling is required in comparison to regular STEMI patients who do not participate in the study. In patients treated with additional MTH due to OHCA it will not be possible to obtain informed consent before blood sampling; however, these patients will require no additional sampling outside the protocol of MTH monitoring. All patients before $\mathrm{pPCl}$ receive a loading dose of aspirin (300 mg) and a loading dose of ticagrelor (180 mg), according to the ESC guidelines.

\section{Study population}

Study group: 25 STEMI patients after OHCA aborted with successful resuscitation, treated with $\mathrm{MTH}, \mathrm{pPCl}$, and ticagrelor.
Control group: 25 STEMI patients treated with $\mathrm{pPCl}$ and ticagrelor (both populations matched regarding the demographic and clinical variables).

Due to the lack of previous relevant studies, the number of patients that allows estimation of the final sample size sufficient to detect statistically significant differences in the primary endpoint was assumed arbitrarily.

Based on a historical analysis of the number of OHCA patients with concomitant STEMI treated with $\mathrm{pPCl}$, who underwent MTH therapy, the time required to enrol the assumed number of patients is estimated to be 20 months.

Inclusion criteria are shown in Table 1, and exclusion criteria are shown in Table 2.

\section{Induction of hypothermia}

The induction of hypothermia will start upon hospital admission, directly after the initial assessment of consciousness [5], and will employ the following:

- ice packs - placed on the largest possible surface area of the patient's body: neck, armpits, abdomen, groin, lower limbs;

- infusion of cold fluid - 1-1.5 $\mathrm{L}$ of $4^{\circ} \mathrm{C}$ lactated Ringer's solution or cold normal saline infused into the peripheral veins;

- washing the patient's skin with alcohol after completion of the diagnostic and invasive procedures, and after final qualification for MTH.

\section{Mild therapeutic hypothermia}

Mild therapeutic hypothermia will be performed and monitored in a Cardiac Intensive Care Unit. The state of $\mathrm{MTH}$ is defined as body core temperature below $34^{\circ} \mathrm{C}$, with a target temperature of $33^{\circ} \mathrm{C}$. To reach the target temperature and maintain it over the subsequent 24 hours, methods of external and intravascular cooling

Table 1. Inclusion criteria

\section{INCLUSION CRITERIA}

\begin{tabular}{ll}
\hline Study group & Control group \\
\hline Age $>18$ years & Age $>18$ years \\
Ecg findings suggestive of STEMI & ECG findings suggestive of stemi \\
$\begin{array}{l}\text { Primary PCl with stenting of at least one coronary artery } \\
\text { Survival from OHCA }\end{array}$ & Primary pci with stenting of at least \\
$\begin{array}{l}\text { Sustained return of spontaneous circulation (ROSC) at least } 20 \text { minutes after the onset } \\
\text { of resuscitation }\end{array}$ & \\
$\begin{array}{l}\text { Unconsciousness (glasgow coma scale score }<8 \text { and }>3 \text { ) after ROSC (in case of prior } \\
\text { sedative drug administration assessed after reversal of their actions with naloxone/anexate) }\end{array}$ & \\
Shockable initial rhythm &
\end{tabular}

ECG - electrocardiography; STEMI — ST-segment elevation myocardial infarction; PCI — percutaneous coronary intervention; OHCA — out-of-hospital cardiac arrest 
Table 2. Exclusion criteria

\section{EXCLUSION CRITERIA}

\section{Study group}

Overt or suspected pregnancy

Known serious infection/sepsis before OHCA

Known bleeding diathesis

Confirmed or suspected internal bleeding

Confirmed or suspected acute stroke

Confirmed or suspected cerebral injury

Known serious neurological dysfunction ( $\mathrm{CPC} \leq 4$ ) before $\mathrm{OHCA}$

Known serious disease with survival prognosis $\leq 180$ days

Haemodynamic instability with systolic blood pressure $<65 \mathrm{~mm} \mathrm{Hg}$ despite treatment

Contraindications to ticagrelor

Administration of glycoprotein Ilb/llla receptor inhibitor

Resuscitation duration $<5$ min

Time delay from ROSC to MTH induction $>240$ minutes

\section{Control group}

Overt or suspected pregnancy

Known serious infection/sepsis before OHCA

Known bleeding diathesis

Confirmed or suspected internal bleeding

Confirmed or suspected acute stroke

Confirmed or suspected cerebral injury

Known serious neurological dysfunction $(\mathrm{CPC} \leq 4)$ before OHCA

Known serious disease with survival prognosis

$\leq 180$ days

Haemodynamic instability with systolic blood pressure

$<65 \mathrm{~mm} \mathrm{Hg}$ despite treatment

Contraindications to ticagrelor

Administration of glycoprotein Ilb/llla receptor inhibitor

$\mathrm{OHCA}$ - out-of-hospital cardiac arrest; CPC — cerebral performance categories; MTH — mild therapeutic hypothermia

will be used. Immediately after $\mathrm{PCl}$ an $\mathrm{MTH}$-dedicated catheter will be placed in the inferior vena cava through the femoral vein under fluoroscopic guidance in the cath lab.

Besides quick arrival at the target temperature and its successful maintenance, properly conducted hypothermia is also characterised by: bradycardia of 40-50 bpm, urine output of $>1 \mathrm{~mL} / \mathrm{kg}$ body weight/hour, and lack of shivers.

The treatment is focused on achieving the designated haemodynamic and biochemical goals. According to the 2008 International Liaison Committee Recommendations, they include [10]:

- body core temperature in the range of $33.0 \pm 0.2^{\circ} \mathrm{C}$;

- mean arterial blood pressure of $65-95 \mathrm{~mm} \mathrm{Hg}$;

- central venous pressure of 8-12 $\mathrm{mm} \mathrm{H}_{2} \mathrm{O}$;

- blood saturation of $92-96 \%(90-94 \%$ for COPD patients);

- diuresis $>1 \mathrm{~mL} / \mathrm{kg}$ body weight $/ \mathrm{h}$;

- temperature-corrected normoxaemia in arterial blood sample;

- temperature-corrected normocapnia in arterial blood sample;

- potassium, calcium, and phosphorus concentrations within normal ranges:

- lactate concentration $<2 \mathrm{mmol} / \mathrm{L}$;

- blood glucose concentration of $110-170 \mathrm{mg} / \mathrm{dL}$;

- maintenance of 24-hour fluid balance (with controlled administration of colloid/crystalloid fluids) at approx. -300 to $0 \mathrm{~mL}$;

- central venous oxygen saturation $>70 \%$.

\section{Pharmacodynamics of ticagrelor}

Blood samples will be drawn at predefined time points: before administration of a 180-mg loading dose of ticagrelor, then after: 30 minutes, and 1, 2, 3, 4, 6 , 12 , and 24 hours.

For assessment of ticagrelor pharmacodynamics four different methods will be used:

- light transmission aggregometry (LTA - 490-4D Chronolog) - ADP-induced aggregation;

- impedance aggregometry using semi-automatic analyser Multiplate - ADP-induced aggregation;

- automatic VerifyNow - P2Y12 assay - ADP-induced aggregation;

- platelet vasodilator-stimulated phosphoprotein (VASP) assay using flow cytometer FASCalibur (the most specific method for the quantification of the pharmacodynamic effects of platelet P2Y12 receptor inhibitors), analysed parameter: platelet reactivity index (PRI/NASP).

\section{Pharmacokinetics of ticagrelor and its active metabolite (AR-C124910XX)}

Blood plasma concentrations of ticagrelor and AR-C124910XX will be evaluated at the same time points as pharmacodynamics, using liquid chromatography mass spectrometry.

\section{Basic study information}

- Single-centre, prospective, observational study;

- comparing two groups of patients:

- OHCA patients after resuscitation undergoing $\mathrm{MTH}$ therapy, treated with primary $\mathrm{PCl}$ and ticagrelor due to STEMI,

- patients with STEMI treated with $\mathrm{pPCl}$ and ticagrelor; 
- duration of observation: 24 hours;

- primary endpoint: ADP-induced platelet aggregation assessed with impedance aggregometry Multiplate analyser at: 30 minutes, and 1, 2, 3, 4, 6, 12, and 24 hours after administration of a 180-mg loading dose of ticagrelor;

- secondary endpoints:

- ADP-induced platelet aggregation assessed with light transmission aggregometry (LTA) at: $30 \mathrm{~min}-$ utes, and 1, 2, 3, 4, 6, 12, and 24 hours after administration of a 180-mg loading dose of ticagrelor.

- platelet reactivity index (PRI/VASP) assessed with flow cytometer at: 30 minutes, and 1, 2, $3,4,6,12$, and 24 hours after administration of a $180-\mathrm{mg}$ loading dose of ticagrelor,

- ADP induced platelet aggregation assessed with VerifyNow analyzer at: 30 minutes, and 1, 2, 3, $4,6,12$, and 24 hours after administration of a 180-mg loading dose of ticagrelor,

- area under the curve of ticagrelor concentration and its metabolite AR-C124910XX levels during follow-up (AUCO- $\tau$ ),

- maximum concentration of ticagrelor $\left(\mathrm{C}_{\max }\right)$ and its metabolite AR-C124910XX,

- time to reach Cmax of ticagrelor and its metabolite AR-C124910XX,

- ticagrelor serum concentration at: 30 minutes, and 1, 2, 3, 4, 6, 12, and 24 hours after administration of a 180-mg loading dose of ticagrelor,

- AR-C124910XX serum concentration at: 30 minutes, and 1, 2, 3, 4, 6, 12, and 24 hours after administration of a 180-mg loading dose of ticagrelor;

- clinical endpoint: stent thrombosis within 24 hours of study;

- safety endpoint: bleeding by the BARC criteria, bradyarrhythmias.

\section{Characteristics of the final result}

Understanding the mechanisms behind the impact of MTH on the efficacy of platelet inhibition with
P2Y12 inhibitors is pivotal to reduce the risk of thrombotic complications. The study is expected to provide information allowing us to improve the safety of MTH in STEMI patients with OHCA, treated with $\mathrm{PPCl}$, and receiving ticagrelor.

This study has been developed as part of the "Diamond Grant" project financed by the Ministry of Science and Higher Education of the Republic of Poland from research funds for the years 2015-2018.

\section{References}

1. Wallentin L, Becker RC, Budaj A et al. Ticagrelor versus clopidogrel in patients with acute coronary syndromes. N Engl J Med 2009; 361: 1045-1057

2. Steg PG, James SK, Atar D et al. ESC Guidelines for the management of acute myocardial infarction in patients presenting with ST-segment elevation. Task Force on the management of ST-segment elevation acute myocardial infarction of the European Society of Cardiology (ESC) Eur Heart J 2012; 33: 2569-2619.

3. O'Gara PT, Kushner FG, Ascheim DD et al. 2013 ACCF/AHA guideline for the management of ST-elevation myocardial infarction: executive summary: a report of the American College of Cardiology Foundation/American Heart Association Task Force on Practice Guidelines. Catheter Cardiovasc Interv 2013; 82: E1-E27.

4. Kozinski M, Pstragowski K, Kubica JM et al. ACS network-based implementation of therapeutic hypothermia for the treatment of comatose out-of-hospital cardiac arrest survivors improves clinical outcomes: the first European experience. Scand J Trauma Resusc Emerg Med 2013; 21: 547-556.

5. Kubica JM, Pstragowski K, Koziński M et al. Milld therapeutic hy pothermia for patients with acute coronary syndrome and cardiac arrest treated with percutaneous coronary intervention. A single center study. Clin Chem Lab Med 2014; 52: eA467- eA468 DOI 10.1515/cclm-2014-0918.

6. Casella G, Carinci V, Cavallo P et al. Combining therapeutic hypothermia and emergent coronary angiography in out-of-hospital cardiac arrest survivors: Optimal post-arrest care for the best patient. Eur Heart J. Acute Cardiovascular Care 12/2014; DOI: $10.1177 / 2048872614564080$

7. Dumas F, White L, Stubbs BA, et al. Long-term prognosis following resuscitation from out of hospital cardiac arrest: role of percutaneous coronary intervention and therapeutic hypothermia. J Am Coll Cardiol 2012; 60: 21-27.

8. Penela $\mathrm{D}$, Magaldi $\mathrm{M}$, Fontanals $\mathrm{J}$ et al. Hypothermia in acute coronary syndrome: brain salvage versus stent thrombosis? J Am Coll Cardiol 2013; 61: 686-687.

9. Ibrahim K, Christoph M, Schmeinck S et al. High rates of prasugrel and ticagrelor nonresponder in patients treated with therapeutic hypothermia after cardiac arrest. Resuscitation 2014; 85: 649-656.

10. Bernard SA, Gray TW, Buist MD et al. Treatment of comatose survivors of out-of-hospital cardiac arrest with induced hypothermia N Engl J Med 2002; 346: 557-563. 